\title{
TRANSITIONING FROM CENTRALIZED TO DISTRIBUTED CONTROL: USING SGAM TO SUPPORT A COLLABORATIVE DEVELOPMENT OF WEB OF CELLS ARCHITECTURE FOR REAL TIME CONTROL
}

\author{
E. Guillo-Sansano, M. H. Syed, P. Dambrauskas, \\ M. Chen, G. M. Burt and S.D.J. McArthur \\ University of Strathclyde, Scotland \\ efren.guillo-sansano@strath.ac.uk
}

\begin{abstract}
This paper shares some early experiences of developing the Web of Cells (WoC) concept for real time implementation supported by the Smart Grid Architecture Model (SGAM) reference framework. One of the use cases identified for the WoC concept is elaborated upon and is mapped to SGAM, providing one of the first examples where the SGAM reference framework has been used to develop a future distributed control architecture for real time implementation in power systems. Furthermore, this paper offers some insight into the key contributions that this approach can bring, such as a more effective interdisciplinary collaboration, better understanding of the control problem, and its implementation and validation.
\end{abstract}

\section{INTRODUCTION}

It is widely recognised that future control concepts for smart grids will require greater use of distributed intelligence, and this leads to increasing complexity in the design and, importantly, the validation of such schemes. The Web of Cells (WoC) architecture proposed by the ELECTRA (European Liaison on Electricity Committed Towards long-term Research Activity) Integrated Research Programme proposes one such future distributed control concept [1].

While the ELECTRA WoC concept is under development; it is essential to identify the pragmatic implications on the business operations, information flows, communication systems and components of the power system that may arise due to the transition from the present day centralized power system architecture to the envisioned distributed power system. The Smart Grid Architecture Model (SGAM) reference framework, a specification of the CENCENELEC-ETSI Smart Grid Coordination Group (SG-CG) through the European Union Mandate M/490, is expected to provide a basis to analyse power system architectures, especially novel developments within power systems. However, the reported use of the SGAM reference framework is limited due to the elaborate process required to formalise new distributed power system concepts into SGAM. Different tools to facilitate the SGAM process are being developed and tested that would provide better use case development, management and visualization, [2].

SGAM spans six hierarchical power system zones (Process, Field, Station, Operation, Enterprise and Market) and five domains (Generation, Transmission, Distribution, Distributed Energy Resources and Customer Premise). Five superimposed layers (Business, Function,
T. Strasser

AIT Austrian Institute of Technology, Austria thomas.strasser@ait.ac.at
Information, Communication and Component Layer) allow for the interoperability in the power system to be depicted. Such a representation enables different smart grid solutions to be compared, tested and validated. As per the resources available in literature [3-5], the procedure of developing a SGAM representation is as follows: All the use cases associated with the system (for example the services of the system) are mapped to the business layer. For each use case, the functionalities that are required are identified and mapped across the domains and zones of the function layer. Next, components are associated to each functionality identified earlier and are populated on the component layer. The information layer in SGAM has two views. In one of the views, also known as the Business Context View, the information that is exchanged between the components is mapped and in the other view, called Canonical View, the data format for the information is mapped. The final layer of SGAM is the communication layer in which the technology used for passing information between components is mapped.

The hierarchical essence of SGAM and the procedure described above, allow for seamless representation of the majority of the solutions for present day centralized power systems. However, there is no guidance available on how distributed concepts or solutions like the WoC can be represented on SGAM. With new distributed solutions for power systems being proposed, the methodology in the literature is no longer directly applicable. In this paper, the use of the SGAM reference framework to develop the WoC concept is explored. A use case identified for the WoC concept is used as the basis for this study. Furthermore, the potential advantages such an approach could offer are discussed.

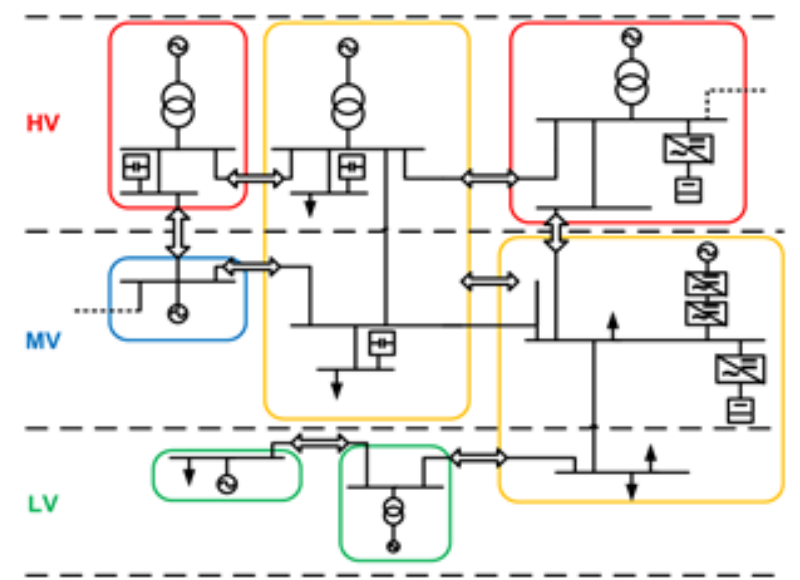

Figure 1: An illustration of the web of cells architecture 


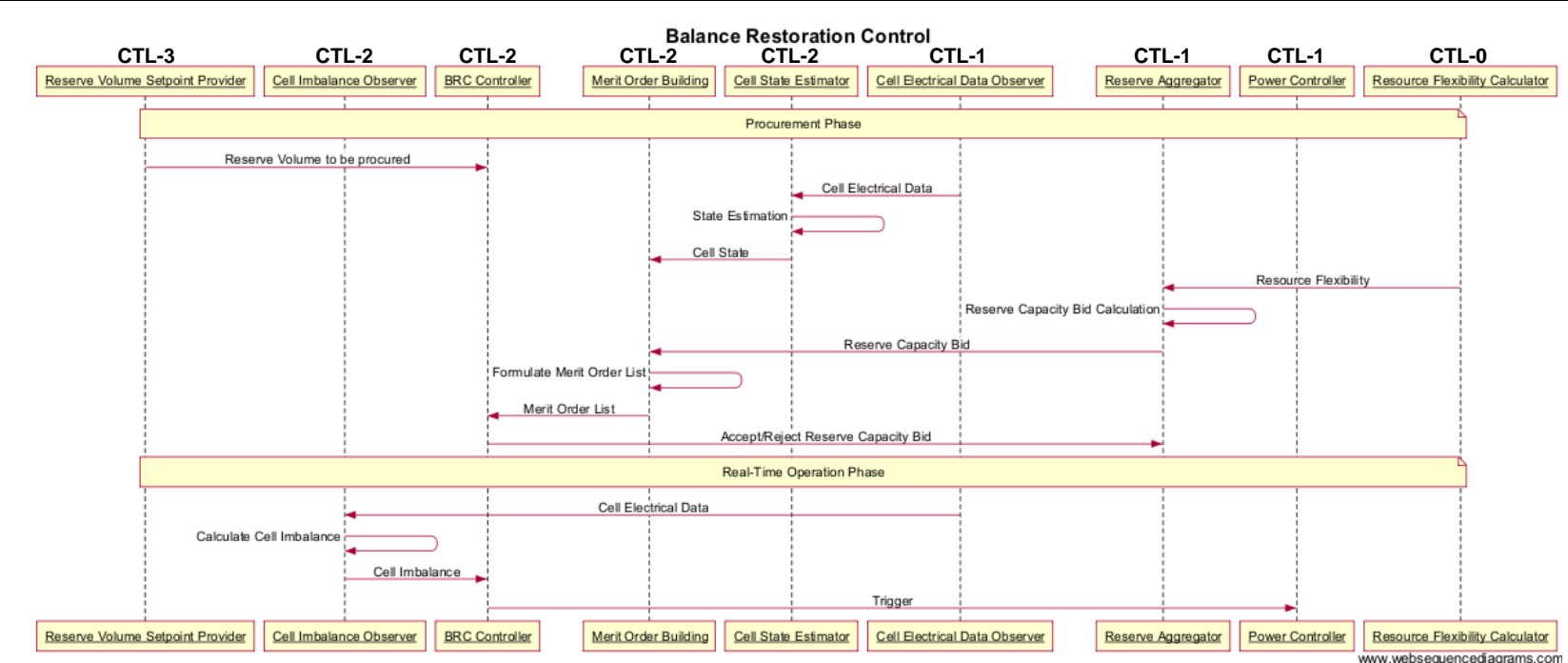

Figure 2: Balance restoration control use case sequence diagram

\section{WOC DEVELOPMENT USING SGAM}

The aim of the WoC architecture is to support a secure system-wide operation by means of distributed control among autonomous regions of the power network, called cells, thereby improving the reliability of future power systems. A cell is defined as "a group of interconnected loads, distributed energy resources and storage units within well-defined grid boundaries corresponding to a physical portion of the grid and to a confined geographical area" [1]. An example of WoC concept is illustrated in Figure 1 where the coloured blocks represent the different cells. Each cell in the network is coequal and no overall system operator with the ability to exercise hierarchical control over cells. The aim here is to achieve a degree of distributed control in real time within the power system. Therefore, it is worthy to mention that distributed control is sought between the different cells in the network while within the cell is hierarchical control.

\section{Use Case Representation on SGAM}

An example use case, Balance Restoration Control (BRC), identified as a key controller within the WoC concept, is presented in Figure 2 in the form of a Unified Modelling Language (UML) sequence diagram. The objective of $\mathrm{BRC}$ is to restore the cell balance, i.e. restore cell consumption/generation to its scheduled value, as well as restore power exchange with other cells to their scheduled values. Compared with ENTSO-E Frequency Restoration Reserve (FRR) [6], ELECTRA definition provides a distributed functionality, and has balance as an explicit objective rather than frequency.

The hierarchical control within the cell is achieved by means of allocating various control functionalities within the cell to four Control Topology Levels (CTLs) [7]. Any control function that exists at a device level (for example a simple local load control like load shedding by relays and circuit breaker) is attributed to CTL-0. A control function at an aggregated level (for example a controller that controls a number of loads like that of a demand side aggregator) is referred to as CTL-1. There might be more than one control for the same function at CTL- 0 and CTL-1. A control function that resides at a cell level is classified as CTL-2. There is only one control per function that can reside at CTL-2. CTL-3 refers to the control functions that are dependent on interactions between the cells.

The various functions with their interactions are identified in accordance with the SGAM procedure along with the CTLs. Although a cell can be well represented on SGAM due to the hierarchical control within it, the question that arises is how a WoC (more than one cell) can be represented on SGAM. As described earlier, there are CTL-3 functions within each cell, there is an exchange of information amongst them, and there is no guidance available on how such interactions can be managed by SGAM. Therefore, this paper proposes an approach that would facilitate the representation of distributed solutions on SGAM.

In order to enable a distributed solution (as the use case in Figure 2 depicts) to be represented on SGAM, the control topology levels defined for the WoC are correlated to equivalent hierarchical zones of SGAM as presented in Table 1. The function layer of SGAM for the BRC use case is presented in Figure 3(a). The simple correlation between the CTLs and SGAM zones enables representation of distributed solutions on SGAM. Any information that is passed from a CTL-3 function to a CTL-2 function can be easily represented just as information flow from any enterprise level function to the operation level function.

A distributed function in a cell requires inputs from within the cell and once acquired can be shared between the cells to make a distributed control decision. It can be assumed

Table 1: CTL Correlation to SGAM Zones
\begin{tabular}{|c|c|}
\hline $\begin{array}{c}\text { Control Topology Level } \\
\text { (CTL) }\end{array}$ & SGAM Zones \\
\hline CTL-0 & Process, Field \\
\hline CTL-1 & Station \\
\hline CTL-2 & Operation \\
\hline CTL-3 & Enterprise \\
\hline
\end{tabular}




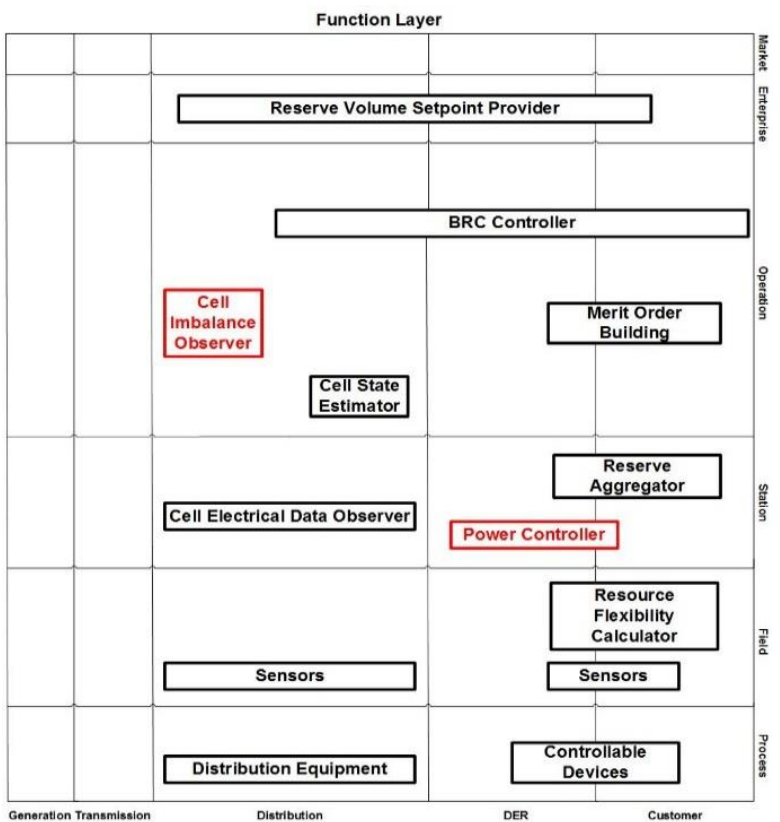

(a)

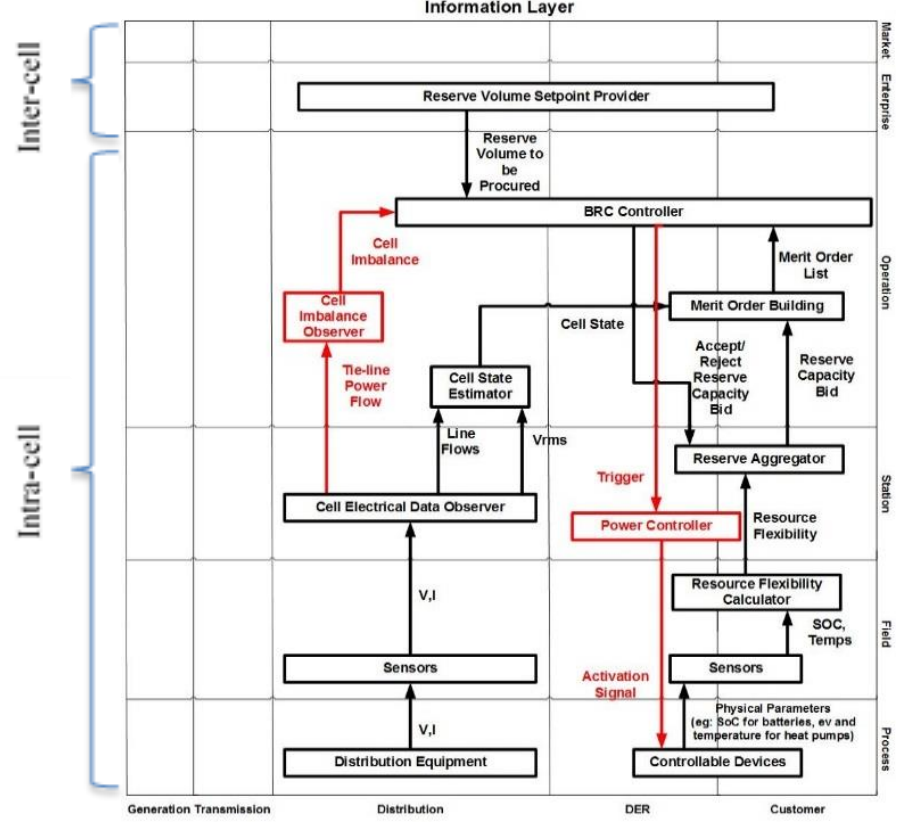

(b) that any input that a CTL-3 function receives from within the cell is shared with neighboring cells in order to reach a distributed decision.

\section{Distributed Solution Development Using SGAM}

In accordance with the above procedure for a particular use case, the component layer is populated after derivation of the function layer. However, for new control solutions that are being developed it would not be appropriate to populate a component layer before the information layer has been developed. This is for the simple reason that while developing new solutions it is important to identify whether or not the existing components present within the system would suffice for the implementation of the new solution. In order for such an analysis to be realized, the information that is exchanged between various functions need to be mapped. Therefore, it is proposed that before the component layer is developed, the information layer is developed and the information that is exchanged is mapped between functions rather than between the components. The proposed business context view of the information layer for the BRC use case is presented in Figure 3(b).

\section{DISCUSSION}

During the SGAM mapping of the WoC distributed power system use case, a number of advantages have been identified from the use of SGAM. A discussion of these advantages encountered is presented in this section.

\section{Scalability}

Using SGAM for the development of new smart grid approaches allows the solutions to be scaled quite easily. As an example, the BRC use case comprises two phases: the procurement phase and the real time control phase.
Once the procurement phase has been mapped to the function layer and the information layer, the team found it easy to add on additional functions thereby extending the model to include the real time control phase. With such an architecture, only the additional information to be exchanged from and to the new functionalities have to be identified and integrated within the existing functions. This could reduce notably the time required for assessing the integration of new functionalities into distributed power systems as well as into legacy centralized ones.

\section{Interdisciplinary Collaboration}

The structured development of superimposed layers in SGAM provides a basis for interdisciplinary collaborative development of new solutions for real time implementation. This improves the communication between different disciplines (e.g. spanning market systems and real time control) and more importantly the efficiency and quality of the development work that can be carried out in a parallel manner as explained in the next section. An example on the interdisciplinary collaboration is shown below for the work that has been presented.

\section{Stakeholders}

The development of a high level use case begins with stakeholders identifying and proposing new solutions in order to either more efficiently utilize the existing grid infrastructure without reinforcements or to meet environmental regulations as targeted by various governments. Once such use cases are identified, they typically form the business layer of SGAM.

\section{Requirements}

With the business layer defined, requirement engineers can interpret a defined use case and identify functional but also non-functional requirements for the use case in order to 
achieve the goals set by the stakeholders. In our experience, the high level use cases need to be very well defined in order to facilitate the rest of the SGAM mapping process.

\section{Control}

Once the business layer has been developed and the requirements have been defined, the control engineers can now develop the control structure necessary to achieve the goal of the high level use case. The functions necessary for the use case can be identified and mapped on to the functional layer. Furthermore, the information that needs to be exchanged between the functions can be defined by the control engineers and represented on the business context view of the information layer. This process has proven to be very challenging due to a lack of familiarity with the SGAM architecture and also to the ambiguity that SGAM zones can bring to the mapping of novel distributed real time control architectures.

\section{Information and Communication}

The business context view of the information layer can now be passed on to the information and communications experts to identify the data formats and the standards that would be necessary for the exchange of information between the functions. This completes the canonical view of the information layer and the communication layer of SGAM.

\section{Parallel Development and Distributed Implementation}

SGAM allows for parallel development of various use cases and the tools that have been developed for SGAM facilitating easy management, and incorporation and integration of various use cases. For the purpose of implementation each use case can be implemented function by function in a distributed manner. For example, the Cell State Estimation function of the BRC use case is dependent upon the Cell Electrical Data Observer and has interactions with the Merit Order Building function as shown in Figure 3. All the other interactions and abundant information available on SGAM is not necessary for its implementation. Hence, all of the information related to Cell State Estimation can easily be extracted and handed to the entity responsible for its implementation.

\section{Testing of Distributed Solutions}

\section{Simulation Prototype Testing}

The two views of the information layer provide sufficient information for developing distributed control prototypes (multi-agent based, service-oriented based, etc.). Such control approaches can be designed to conform to the requirements of the use case and to the data formats as prescribed on the information layer. This allows for a fast prototype development for early simulation stages.

\section{Laboratory Validation}

Before any solution can be implemented in the real-world, the proposed solution needs to be validated by means of laboratory tests (incl. real-time simulations and hardware-inthe-loop experiments). Possessing the function layer, the information layer and the communication layer of SGAM allow different laboratories to design and conduct interlaboratory experiments, multiple times, independently. This early stage testing on laboratory contributes to de-risking and refining future real time control concepts.

\section{Real-World Implementation}

Once a new control solution has been validated and tested in laboratories, the power engineers can now decide upon the components in the network that would enable the field implementation of the new solution and hence provide the final component layer of SGAM.

\section{CONCLUSIONS}

In this paper, the use of SGAM for the development of a distributed solution for real time control has been presented. The experience gained throughout this process has contributed to present a different procedure for the mapping of the distributed concept. Such an approach is helpful for the interdisciplinary collaboration between different domains (power system, information and control system), the control concept development itself but also for the necessary validation and testing.

\section{ACKNOWLEDGEMENT}

The work in this paper has been in part supported by the European Commission under the FP7 project ELECTRA IRP (grant no: 609687). Any opinions, findings and conclusions or recommendations expressed in this material are those of the authors and do not necessarily reflect those of the European Commission. It has also been part funded through EPSRC HubNet (grant ref: EP/I013636/1).

\section{REFERENCES}

[1] L. Martini, L. Radaelli, H. Brunner, C. Caerts, A. Morch, S. Hanninen, and C. Tornelli, 2015, "ELECTRA IRP approach to voltage and frequency control for future power systems with high DER penetrations", Proceedings of $23^{\text {rd }}$ International Conference on Electricity Distribution (CIRED), Lyon.

[2] SGAM Toolbox, en-trust, Josef Ressel Center for User-Centric Smart Grid Privacy, Security and Control, [Online] Available: http://www.en-trust.at/downloads/sgam-toolbox/

[3] C. Neureiter, D. Engel, J. Trefke, R. Santodomingo, S. Rohjans and, M. Uslar, "Towards consistent smart grid architecture tool support: From use cases to visualization," Proceedings of 2014 IEEE PES Innovative Smart Grid Technologies Conference Europe (ISGTEurope), Istanbul.

[4] Smart Grid Coordination Group (SG-CG) Reference Architecture Smart Grid, CEN, CENELEC, and ETSI, Nov. 2012.

[5] R. Santodomingo, M. Uslar, A. Goring, M. Gottschalk, L. Nordstrom, A. Saleem, and M. Chenine, 2014, "SGAM-based methodology to analyse Smart Grid solutions in DISCERN European research project", Proceedings of 2014 IEEE International Energy Conference (ENERGYCON), Dubrovnik.

[6] Supporting Document for the Network Code on Load-Frequency Control and Reserves, ENTSO-E, [Online] Available: https://www.entsoe.eu/fileadmin/user_upload/ library/resources/LCFR/ 130628-NC LFCR-Supporting Document-Issue1.pdf

[7] K. Heussen, M. Uslar, and C. Tornelli, 2015, "A use case methodology to handle conflicting controller requirements for future power systems," Proceedings of 2015 International Symposium on Smart Electric Distribution Systems and Technologies (EDST), Vienna. 